node (LN) status. LN assessment by imaging methods has several known limitations including a high false negative rate. The present study aimed to compare the accuracy of LN staging by imaging and surgical staging in LACC patients, and to evaluate their impact on radiation field planning.

Methodology A retrospective monocentric study of patients with LACC (International Federation of Gynecology and Obstetrics (FIGO 2018) stage IIA -IVA), undergoing primary definitive platinum-based chemoradiation therapy. Patients were included if $\mathrm{LN}$ assessment was available by both methods: surgical (paraaortic/pelvic) and imaging [Thorax/Abdomen Computed Tomography (CT) and/or pelvic Magnetic Resonance Imaging (MRI)].

Result(s)* A total of 58 patients met the inclusion criteria (table 1), 97\% $(\mathrm{n}=56)$ had a preoperative CT and $88 \%$ $(\mathrm{n}=51)$ an MRI evaluation. All patients underwent surgical LN staging: $100 \%$ paraaortic, and $86 \%(n=50)$ additional pelvic lymphadenectomy. Histologically proven LN metastases after surgical LN staging were found in $76 \%$ of patients $(n=44), 31 \%(n=18)$ paraaortic and $76 \%(n=38)$ pelvic. As a result of the surgical LN staging, 36\% $(n=21)$ of the patients were upstaged $(n=11$ to FIGO IIIC1 and $n=10$ to FIGO IIIC2), and $17 \%(n=10)$ had treatment modification (extended paraaortic field radiation). LN staging using CT and MRI exhibited a low negative predictive value $(29 \%$ and $38 \%$, respectively), with a higher positive predictive value $(69 \%$ and $81 \%$, respectively).

Conclusion* In this cohort of LACC patients, paraaortic LN metastases were present in one third of the cases, while CT/ MRI imaging underestimated metastatic LN involvement. We thus stress the value of surgical paraaortic LN staging in cases of negative LN imaging, which may lead to treatment modification in about one fifth of patients.

\section{THE IMPACT OF MICROMETASTASES IN CERVICAL CANCER PATIENTS - A RETROSPECTIVE STUDY OF THE SCCAN (SURVEILLANCE IN CERVICAL CANCER) PROJECT}

${ }^{1} \mathrm{~L}$ Dostalek*, ${ }^{1} \mathrm{M}$ Borcinova, ${ }^{2} \mathrm{~K}$ Benesova, ${ }^{3} \mathrm{~J}$ Klat, ${ }^{4} \mathrm{H}$ Falconer, ${ }^{5} \mathrm{SH}$ Kim, ${ }^{6} \mathrm{LR}$ Van Lonkhuijzen, ${ }^{7} \mathrm{~A}$ Lopez, ${ }^{8} \mathrm{D}$ Isla Ortiz, ${ }^{9} \mathrm{~F}$ Landoni, ${ }^{10}$ J Kostun, ${ }^{11} \mathrm{R}$ Dos Reis, ${ }^{12} \mathrm{D}$ Odetto, ${ }^{13} \mid$ Zapardiel, ${ }^{2} \mathrm{~J} J a r k o v s k y,{ }^{3} \mathrm{~V}$ Javukova, ${ }^{4} \mathrm{~S}$ Salehi, ${ }^{5} \mathrm{NR}$ Abu-Rustum, ${ }^{3} \mathrm{P}$ Graf, ${ }^{1} \mathrm{D}$ Cibula. ${ }^{1}$ Gynecologic Oncology Center, Department of Obstetrics and Gynecology, First Faculty of Medicine, Charles University and General University Hospital, Central and Eastern European Gynecologic Oncology Group, (CEEGOG), Prague, Czech Republic; ${ }^{2}$ Institute of Biostatistics and Analyses, Faculty of Medicine, Masaryk University, Brno, Czech Republic; ${ }^{3}$ Department of Obstetrics and Gynecology, Faculty of Medicine, University Hospital and University of Ostrava, Ostrava , Czech Republic; ${ }^{4}$ Department of Pelvic Cancer, Karolinska University Hospital and Department of Women's and Children's Health, Karolinska Institutet, Stockholm, Sweden; ${ }^{5}$ Department of Surgery, Memorial Sloan Kettering Cancer Center, New York, NY 10065, USA; ${ }^{6}$ Department of Gynecological Oncology, Amsterdam University Medical Center-Center for Gynecological Oncology Amsterdam, Amsterdam, the Netherlands; 'Department of Gynecological Surgery, National Institute of Neoplastic Diseases, Lima, Peru; ${ }^{8}$ Gynecology Oncology Center, National Institute of Cancerology Mexico, Mexico; ${ }^{9}$ University of Milano-Bicocca,Department of Obstetrics and Gynecology, Gynaecologic Oncology Surgical Unit, ASST-Monza, San Gerardo Hospital, Monza, Italy; ${ }^{10}$ Department of Gynaecology and Obstetrics, University Hospital Pilsen, Charles University, Prague, Czech Republic; "11Departamento de Ginecologia Oncológica, Hospital de Amor Barretos, Brazil; ${ }^{12}$ Department of Gynecologic Oncology , Hospital Italiano de Buenos Aires, Instituto Universitario Hospital Italiano, Buenos Aires, Argentina; ${ }^{13}$ Gynecologic Oncology Unit, La Paz University Hospital - IdiPAZ, Madrid, Spain
Introduction/Background* The impact of lymph node (LN) micrometastases (MIC) in cervical cancer patients remains a controversial topic given their low incidence and good prognosis of patients managed by primary surgery.

We aim to evaluate the prognostic significance of MIC and isolated tumour cells (ITC) in a large cohort of patients from the SCCAN retrospetive study (Surveillance in Cervical CANcer). SCCAN study analysed data from more than 4300 patients with early stage cervical cancer treated by primary surgery at 20 large tertiary institutions from Europe, North America, South America and Australia.

Methodology In this SCCAN sub-study, we included patients with early stage cervical cancer (T1a1 LVSI+ - T2b) treated between 2007 and 2016 with at least 1-year follow-up data availability, who underwent primary surgery including sentinel lymph node (SLN) biopsy and in whom SLNs were processed by pathological ultrastaging protocol.

Abstract 898 Table 1 Data summary $(\mathrm{N}=969)$

\begin{tabular}{|c|c|c|}
\hline Characteristics & & Description \\
\hline \multirow[t]{3}{*}{ Tracer type } & Radiocolloid & $423(43.7 \%)$ \\
\hline & Dye & $662(68.3 \%)$ \\
\hline & ICG & $220(22.7 \%)$ \\
\hline No. of SLN detected & Mean \pm SD & $3.2 \pm 2.2$ \\
\hline \multirow{4}{*}{$\begin{array}{l}\text { Largest type of metastasis in LN } \\
\text { including SLN }\end{array}$} & Negative & $795(82.0 \%)$ \\
\hline & ITC & $31(3.2 \%)$ \\
\hline & MIC & $59(6.1 \%)$ \\
\hline & MAC & $84(8.7 \%)$ \\
\hline \multirow[t]{3}{*}{ Surgical approach } & Open & $575(59.3 \%)$ \\
\hline & Robotic & $195(20.1 \%)$ \\
\hline & Laparoscopic & $199(21.5 \%)$ \\
\hline \multirow[t]{5}{*}{ Tumour histotype } & Squamous & $605(62.4 \%)$ \\
\hline & Adenocarcinoma & $287(29.6 \%)$ \\
\hline & Adenosquamous & $50(5.2 \%)$ \\
\hline & Neuroendocrine & $18(1.9 \%)$ \\
\hline & Other & $9(0.9 \%)$ \\
\hline \multirow[t]{4}{*}{ Grade } & 1 & $149(15.4 \%)$ \\
\hline & 2 & $406(41.9 \%)$ \\
\hline & 3 & $246(25.4 \%)$ \\
\hline & N/A & $168(17.3 \%)$ \\
\hline \multirow[t]{3}{*}{ LVSI } & No & $316(32.6 \%)$ \\
\hline & Yes & $351(36.2 \%)$ \\
\hline & $N / A$ & $302(31.2 \%)$ \\
\hline \multirow{6}{*}{$\begin{array}{l}\text { Maximal pathologic tumour diameter } \\
{[\mathrm{mm}]}\end{array}$} & Mean \pm SD & $20.6 \pm 13.7$ \\
\hline & Median (IQR) & $19(10 ; 30)$ \\
\hline & $<0.5 \mathrm{~cm}$ & $73(7.5 \%)$ \\
\hline & $0.5-1.99 \mathrm{~cm}$ & $424(43.8 \%)$ \\
\hline & $2-3.99 \mathrm{~cm}$ & $376(38.8 \%)$ \\
\hline & $\geq 4 \mathrm{~cm}$ & $96(9.9 \%)$ \\
\hline Adjuvant therapy & & $312(32.2 \%)$ \\
\hline \multirow[t]{4}{*}{ if yes: } & radiotherapy & $153(49.0 \%)$ \\
\hline & chemoradiotherapy & $136(43.6 \%)$ \\
\hline & chemotherapy & $18(5.8 \%)$ \\
\hline & $\begin{array}{l}\text { chemoradiotherapy + } \\
\text { chemotherapy }\end{array}$ & $5(1.6 \%)$ \\
\hline
\end{tabular}

Recurrence

$117(12.1 \%)$ 
Disease-free survival: All patients by largest type of metastasis in LN ( $N=969)$

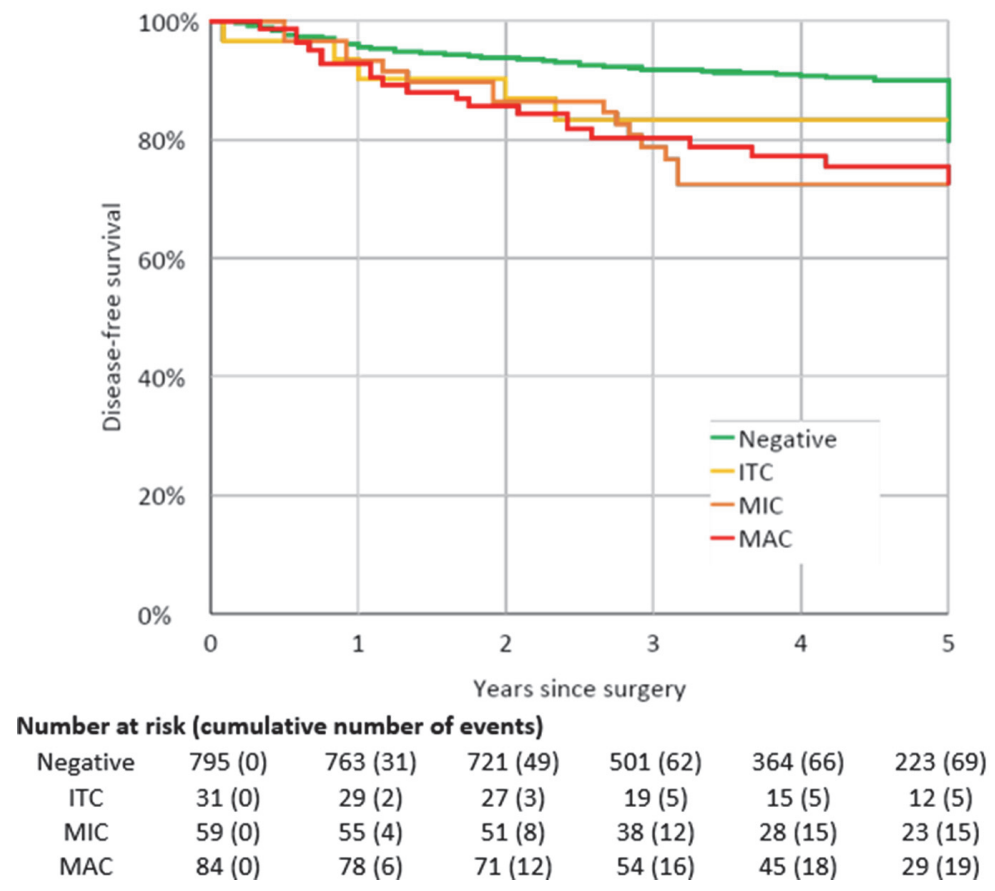

Abstract 898 Figure 1

Abstract 898 Table 2 Univariate analysis of factors associated with disease-free survival $(\mathrm{N}=969)$

\begin{tabular}{|c|c|c|c|c|}
\hline Predictor & Category & $\mathrm{n}$ & $\mathrm{HR}(95 \% \mathrm{Cl})$ & p-value \\
\hline \multirow[t]{4}{*}{ Surgical approach } & Open & 575 & Ref. & \\
\hline & Robotic & 195 & $1.21(0.74 ; 1.97)$ & 0.439 \\
\hline & Laparoscopic & 141 & $1.51(0.93 ; 2.45)$ & 0.097 \\
\hline & Combined & 58 & $1.06(0.48 ; 2.31)$ & 0.888 \\
\hline \multirow[t]{4}{*}{ Tumour diameter } & $<0.5 \mathrm{~cm}$ & 73 & Ref. & \\
\hline & $0.5-1.99 \mathrm{~cm}$ & 424 & $1.67(0.51 ; 5.47)$ & 0.399 \\
\hline & $2-3.99 \mathrm{~cm}$ & 376 & $3.98(1.25 ; 12.69)$ & 0.019 \\
\hline & $\geq 4 \mathrm{~cm}$ & 96 & $6.35(1.91 ; 21.13)$ & 0.003 \\
\hline \multirow[t]{2}{*}{ LVSI } & No & 316 & Ref. & \\
\hline & Yes & 351 & $2.31(1.47 ; 3.63)$ & $<0.001$ \\
\hline \multirow[t]{4}{*}{ Tumour histotype } & Squamous & 605 & Ref. & \\
\hline & Adenocarc. & 287 & $1.13(0.75 ; 1.71)$ & 0.554 \\
\hline & Adenosquamous & 50 & $1.38(0.66 ; 2.89)$ & 0.385 \\
\hline & Other & 27 & $3.03(1.45 ; 6.31)$ & 0.003 \\
\hline \multirow[t]{3}{*}{ Grade } & 1 & 149 & Ref. & \\
\hline & 2 & 406 & $2.08(1.02 ; 4.22)$ & 0.044 \\
\hline & 3 & 246 & $3.35(1.64 ; 6.85)$ & $<0.001$ \\
\hline \multirow{4}{*}{$\begin{array}{l}\text { Largest type of } \\
\text { metastasis in LN }\end{array}$} & Negative & 795 & Ref. & \\
\hline & ITC & 31 & $1.67(0.68 ; 4.14)$ & 0.264 \\
\hline & MIC & 59 & $2.55(1.47 ; 4.43)$ & $<0.001$ \\
\hline & MAC & 84 & $2.36(1.44 ; 3.87)$ & $<0.001$ \\
\hline \multirow{3}{*}{$\begin{array}{l}\text { Largest type of } \\
\text { metastasis in LN }\end{array}$} & Negative & 795 & Ref. & \\
\hline & ITC & 31 & $1.67(0.68 ; 4.14)$ & 0.264 \\
\hline & $\mathrm{MIC}+\mathrm{MAC}$ & 143 & $2.44(1.63 ; 3.64)$ & $<0.001$ \\
\hline
\end{tabular}

Result(s)* Out of 969 included patients with at least 1 SLN detected, $174(18 \%)$ had positive LN (table 1). Maximal tumour diameter $>2 \mathrm{~cm}$, positive LVSI, grade $\geq 2$, uncommon histological type (neuroendocrine, sarcoma, etc.) and macrometstasis (MAC) or MIC in LN were factors associated with significantly decreased five-years disease free survival (DFS) (table 2). MAC, MIC or ITC was the largest LN metastasis in 84 (9\%), $59(6 \%)$ and $31(3 \%)$ cases respectively. Adjuvant (chemo)radiation was administred in $89 \%, 85 \%$ and $58 \%$ of patients with MAC, MIC and ITC. DFS reached 75\%, 73\% and $83 \%$ in patients with MAC, MIC and ITC compared with $90 \%$ in the N0 patients. Patients with MAC and MIC had significantly decreased DFS than those with N0 disease $(\mathrm{HR}=2.36$ and 2.55).

Conclusion* Early-stage cervical cancer patients with MIC in pelvic LN have significantly decreased DFS. Their management should follow the same principles as in patients with MAC.

\section{PHASE 1B TRIAL OF FIRST-LINE BINTRAFUSP ALFA, A BIFUNCTIONAL FUSION PROTEIN TARGETING TGF- $\beta$ AND PD-L1, PLUS CHEMOTHERAPY WITH OR WITHOUT BEVACIZUMAB IN CERVICAL CANCER}

${ }^{1} \mathrm{~A}$ Oaknin*, ${ }^{2} \mathrm{M}$ Gil-Martin, ${ }^{3} \mathrm{E}$ Diver, ${ }^{4} \mathrm{G}$ Jehl, ${ }^{4} \mathrm{SA}$ Gleicher, ${ }^{5} \mathrm{~S}$ Chaudhary, ${ }^{5} \mathrm{~L}$ Ojalvo, ${ }^{6} \mathrm{~K}$ Hasegawa. 'Vall d'Hebron University Hospital, Vall d'Hebron Institute of Oncology (VHIO), Barcelona, Spain; ${ }^{2}$ ICO Hospital Duran i Reynals, Barcelona, Spain; ${ }^{3}$ Stanford Cancer Institute, Stanford, CA, USA; ${ }^{4}$ Merck KGaA, Darmstadt, Germany; ${ }^{5}$ EMD Serono Research and Development Institute, Inc., Billerica, MA, USA; ${ }^{6}$ Saitama Medical University International Medical Center, Hidaki-shi, Saitama-ken, Japan 